\title{
Delayed Eruption of Permanent Lower Lateral Incisor in Relation to Compound Odontoma: A Case Report
}

\author{
Omar A Bawazir
}

\begin{abstract}
Aim: To report a delayed tooth eruption following late surgical removal of a compound odontoma in a 10-year-old child.

Background: Odontomas are the most common odontogenic tumors. They are usually asymptomatic and discovered during routine radiographic examinations or during an assessment of delayed tooth eruption. Odontomas are classified into compound or complex based on the microscopic structure of dental tissues. Early diagnosis and surgical removal of odontomas is the keyword for favorable results.

Case description: This paper describes a case of compound odontomas in a 10-year-old boy that prevented eruption of the permanent mandibular lateral incisor. Extraction of mandibular primary lateral incisors and surgical excision of the compound odontomas was performed. After 3 years of follow-up, at the age of 13 years, the permanent lateral incisor erupted and approached the occlusal plane.

Conclusion: Late surgical removal of odontomas resulted in a delayed eruption of the affected teeth.

Clinical significance: Surgical removal of odontomas and regular follow-up to monitor the eruption of the affected teeth should be considered for the teeth with an open apex before considering orthodontic traction.

Keywords: Compound odontoma, Delayed eruption, Odontogenic tumor.

The Journal of Contemporary Dental Practice (2021): 10.5005/jp-journals-10024-3155
\end{abstract}

\section{INTRODUCTION}

The term "odontomas" is applied to a pathologic hamartoma of odontogenic origin. ${ }^{1}$ Odontomas are the most common benign odontogenic tumors of epithelial and mesenchymal origin and constitute about $22 \%$ of all odontogenic tumors of the jaws. ${ }^{1}$ Recent WHO classification of head and neck tumors (2017) classified odontomas as compound or complex type. ${ }^{2}$ They are composed of mature enamel, dentin, and pulp and may be termed as "compound" or "complex," depending on the extent of morphodifferentiation or their resemblance to normal teeth. ${ }^{1,3}$

The etiology of odontomas is unknown; however, it has been suggested that genetic factors, family history, hyperactivity of odontoblast cells, and local factors, such as trauma or infection, can play a role in its appearance. ${ }^{1,3,4}$ Odontomas are usually asymptomatic and diagnosed during a routine radiographic examination or the evaluation of the cause of delayed tooth eruption. ${ }^{5}$ Majority of the studies reported detection of odontomas during the first two decades of life and most of them being associated with unerupted teeth. . $^{3,6-9}$

The most common pathological changes associated with odontomas are impaction, displacement, resorption, delayed eruption of adjacent teeth or cystic formation, and rarely showed absence of teeth. ${ }^{3,10}$ Odontomas are usually managed by surgical excision with a less likely chance of recurrence. ${ }^{11}$ The management of adjacent teeth is mainly depending on the time of the detection of odontomas, the extent of the damage to adjacent teeth, the position and stage of tooth development, and the space available in the arch. ${ }^{12}$ Regular clinical and radiographic monitoring for a spontaneous eruption of adjacent teeth following removal of odontomas has been reported to be predictable; however, orthodontic traction can be warranted for cases failing to erupt or to correct the tooth position. ${ }^{13}$ This case report showed delayed
Department of Pediatric Dentistry and Orthodontics, College of Dentistry, King Saud University, Riyadh, Saudi Arabia

Corresponding Author: Omar A Bawazir, Department of Pediatric Dentistry and Orthodontics, College of Dentistry, King Saud University, Riyadh, Saudi Arabia, e-mail: obawazir@ksu.edu.sa

How to cite this article: Bawazir OA. Delayed Eruption of Permanent Lower Lateral Incisor in Relation to Compound Odontoma: A Case Report. J Contemp Dent Pract 2021;22(9):1060-1062.

Source of support: Nil

Conflict of interest: None

eruption of permanent lower lateral incisors following late surgical removal of compound odontomas.

\section{Case Description}

An 8-year-old boy presented to the pediatric dental clinics in April 2015 with a complaint of crowded lower incisors. Past medical history was unremarkable. Clinical examination revealed poor oral hygiene, mixed dentition, Angle's class I malocclusion, molar incisors hypomineralization of the lower first permanent molars and upper left central incisor. In the mandible, slight crowding was noticed in the incisor area and the following teeth were present: permanent central and right lateral incisors and retained lower primary left lateral incisor. A panoramic and periapical X-ray showed a radiopaque mass below the root of the lower primary left lateral incisor that was displaced and prevented the eruption of the lower permanent left lateral incisor (Fig. 1). The initial diagnosis based on the clinical and radiographic findings was a compound odontoma. The findings and the plan for surgical excision of the mass were explained to the parents who failed to comply with the

(0) The Author(s). 2021 Open Access This article is distributed under the terms of the Creative Commons Attribution 4.0 International License (https://creativecommons. org/licenses/by-nc/4.0/), which permits unrestricted use, distribution, and non-commercial reproduction in any medium, provided you give appropriate credit to the original author(s) and the source, provide a link to the Creative Commons license, and indicate if changes were made. The Creative Commons Public Domain Dedication waiver (http://creativecommons.org/publicdomain/zero/1.0/) applies to the data made available in this article, unless otherwise stated. 

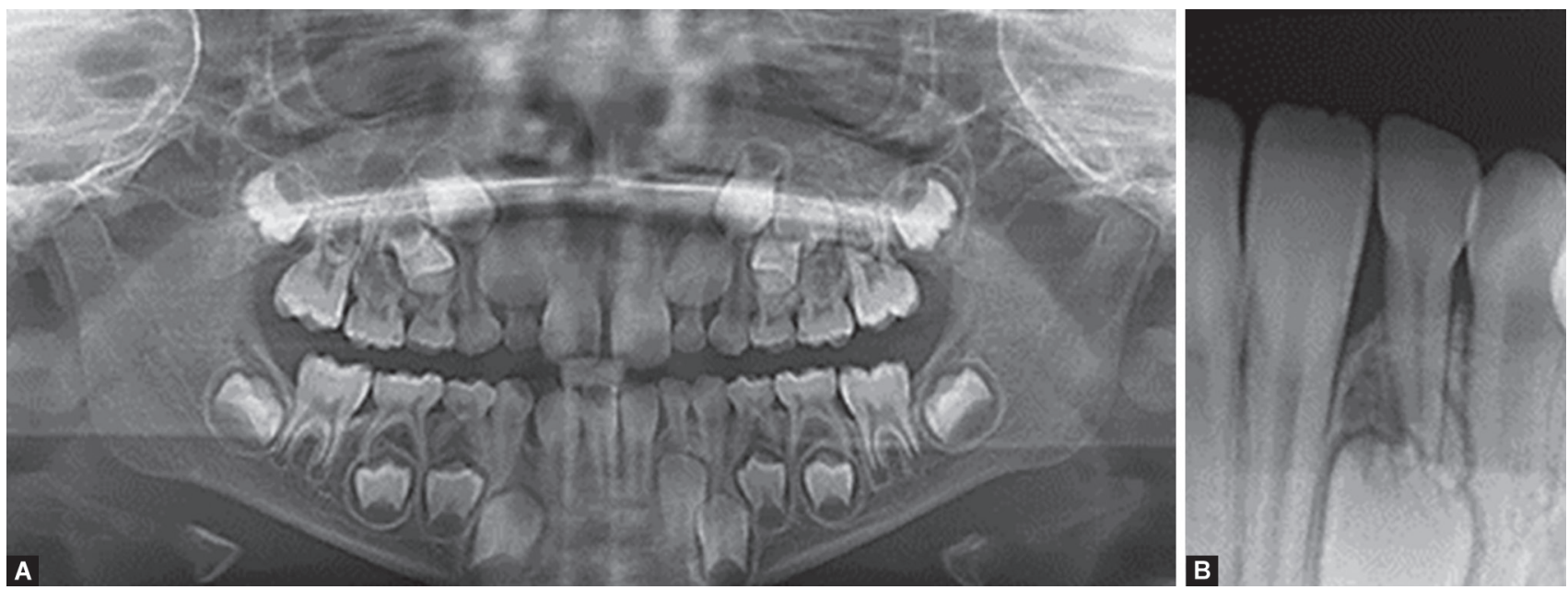

Figs $1 \mathrm{~A}$ and B: Panoramic and periapical radiographs at the age of 8 years showing the compound odontoma obstructing the eruption of the permanent lower left lateral incisor

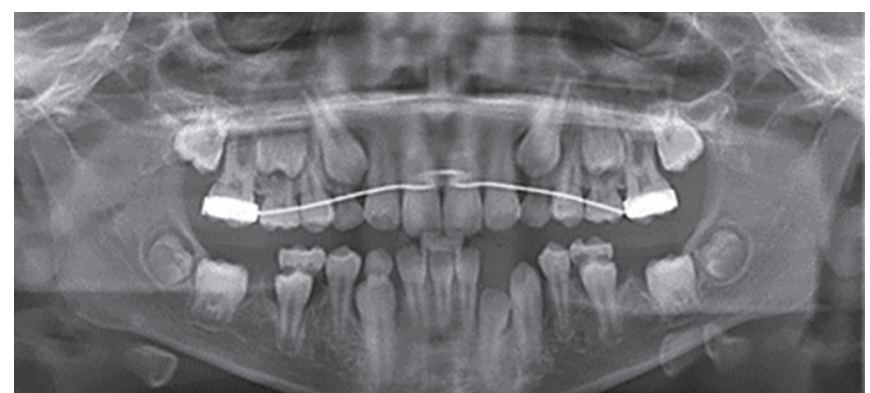

Fig. 2: Panoramic radiograph at the age of 11 years showing improvement of the position of the permanent lower left lateral incisor

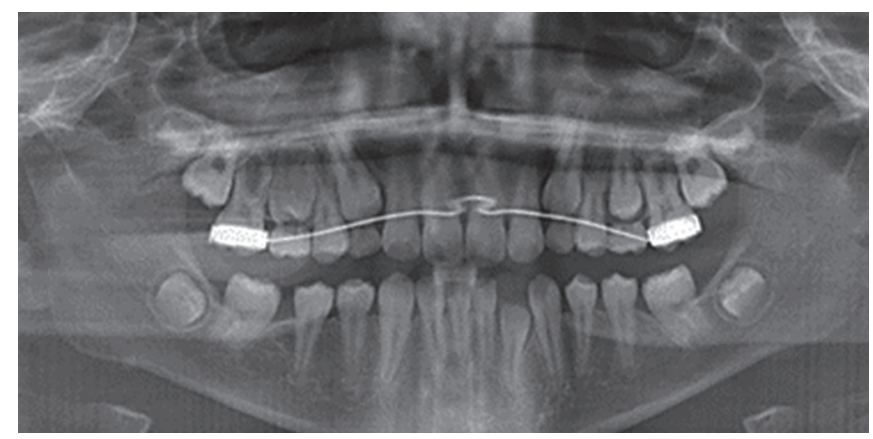

Fig. 3: Panoramic radiograph at the age of 12 years showing further eruption of permanent lower left lateral incisor that was covered with soft tissue

proposed plan immediately. In April 2017, at the age of 10 years, the child came with his parents seeking continuity of the proposed treatment plan. The clinical examination showed no significant changes except that the lower left primary canine became loose and the lower first permanent molars were grossly decayed and indicated for extraction. The plan was revised with the parents and the child was treated with extraction of the lower left primary lateral incisors and loose primary canines followed by widening of the socket and surgical excision of the odontoma under local anesthesia. There were $13 \mathrm{mini}$ tooth-like structures, hard in consistency with variable sizes that were sent for histopathological

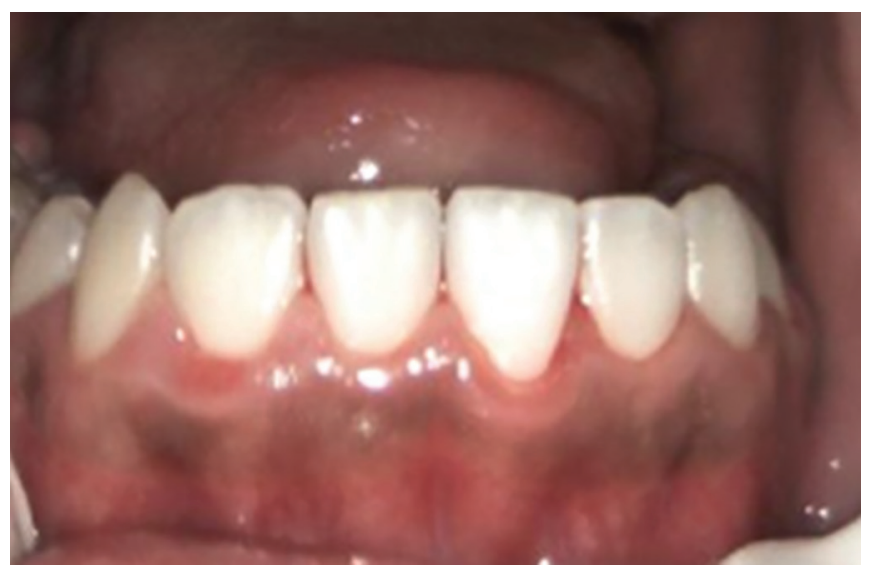

Fig. 4: Clinical photograph at the age of 13 years showing fully erupted permanent lower left lateral incisor into its normal position

examination. The histopathological examination of the mass showed hard well-arranged dental tissues composed of enamel, dentin, and pulp tissues that were consistent with a compound odontoma. Postoperative healing was uneventful. In October 2018, at the age of 11 years, the position of the permanent lower left incisor in the panoramic radiograph was improved (Fig. 2) and in September 2019, the tooth near eruption was covered only by soft tissue (Fig. 3). In August 2020, at the age of 13 years, the lower left lateral incisor was fully erupted into its normal position without any orthodontic traction (Fig. 4).

\section{Discussion}

Odontomas are generally diagnosed during a routine radiographic evaluation and appear as small, solitary, or multiple radiopaque lesions surrounded by a radiolucent zone and separated from the bone by a well-corticated line. ' Compound odontomas appear in the radiograph as multiple irregular radiopaque lesions that vary in size and shape called denticles, while complex odontomas present as a radiopaque solid mass with occasional nodular elements. ${ }^{1,5}$

Compound odontomas are commonly located in the anterior region of the maxilla, while complex odontomas are generally located in the posterior area of the mandible. ${ }^{6-9,14}$ Studies have 
shown a correlation between patient's age and the type of odontomas where compound odontomas are diagnosed at a younger age than complex odontomas. ${ }^{14,15}$

The diagnosis of odontomas is usually based on the clinical and radiographic examination and confirmed by a histopathological assessment of the specimen. ${ }^{1,2,11}$ Delayed tooth eruption is the most common sign associated with odontomas. ${ }^{7,14,16}$ Early detection and surgical removal of odontomas resulted in favorable outcomes and spontaneous eruption of adjacent teeth is expected..$^{11,12}$

In the present case, the child came for an assessment of simple crowding in the lower incisors which showed delayed eruption of the permanent lower left lateral incisor. The routine radiographic assessment revealed the presence of an odontoma that prevented the eruption of the tooth. This scenario is appropriate with previous reports. ${ }^{5,7,14,16}$

A surgical intervention was planned at age 8; unfortunately, the patient did not show up for the scheduled appointment. After 2 years and at the age of 10 years, the surgical intervention was conducted. The generally accepted techniques for the management of odontomas are surgical excision, orthodontic traction, or periodic clinical and radiographic evaluation. $6,13,15,16$ The eruption of the permanent lateral incisors into its normal position at age 13 and after 3 years from surgical intervention is considered unusual. The possible explanation for this is the delayed root formation and open apex of the lower permanent lateral incisor plus the presence of sufficient space in the arch. A similar result of delayed eruption of upper lateral following surgical removal of the compound odontoma was reported and attributed the delayed eruption closely related to the root formation. ${ }^{17}$

\section{Conclusion}

The present case shows successful management of a compound odontoma with minimal surgical intervention and regular follow-up over 3 years until the permanent lateral incisor erupts into its normal position. Delayed eruption of the affected tooth following late surgical removal of a compound odontoma was noticed. Removal of odontoma and monitoring the eruption of the affected teeth should be considered for the teeth with open apex before considering further surgical and orthodontic intervention.

\section{Consent for Publication}

Written informed consent was obtained from the patient for publication of this case report and any accompanying images.

\section{References}

1. Shafer WG, Hine MKLB. Cysts and tumors of the jaws. In: A textbook of oral pathology. 5th ed. Philadelphia:WB Saunders Co; 2005. p. 305-317.
2. El-Naggar AK, Chan JKC, Grandis JR. WHO classification of head and neck tumours. In Lyon: IARC; 2017. p. 204-260.

3. Budnick SD. Compound and complex odontomas. Oral Surg Oral Med Oral Pathol 1976;42(4):501-506. DOI: 10.1016/00304220(76)90297-8. Available from: https://linkinghub.elsevier.com/ retrieve/pii/0030422076902978.

4. Hitchin AD. The aetiology of the calcified composite odontomes. $\mathrm{Br}$ Dent J 1971;130(11):475-482. DOI: 10.1038/sj.bdj.4802682. Available from: http://www.nature.com/articles/4802682.

5. Patil S, Rahman F, Tipu SR, et al. Odontomas: review of literature and report of a case. Oral Maxillofac Pathol J 2012;3(1):1-4. Available at: http://ompj.org/files/aeb864997d1ee10797b3641d8e225820Santosh.pdf

6. Katz RW. An analysis of compound and complex odontomas. ASDC J Dent Child 1989;56(6):445-449. Available at: https://www.aapd.org/ globalassets/media/publications/jdc-archives/asdc-nov-dec-1989v56no6.pdf

7. Owens BM, Schuman NJ, Mincer HH, et al. Dental odontomas: a retrospective study of 104 cases. J Clin Pediatr Dent 1997;21(3): 261-264. Available at: https://meridian.allenpress.com/jcpd

8. Tomizawa M, Otsuka Y, Noda T. Clinical observations of odontomas in Japanese children: 39 cases including one recurrent case. Int J Paediatr Dent 2005;15(1):37-43. DOI: 10.1111/j.1365263X.2005.00607.x.

9. An SY, An CH, Choi KS. Odontoma: a retrospective study of 73 cases. Imaging Sci Dent 2012;42(2):77-81. DOI: 10.5624/isd.2012.42.2.77.

10. Lee $\mathrm{CH}$, Park GJ. Complex and compound odontomas are clinicopathological entities. Basic Appl Pathol 2008;1(1):30-33. DOI: 10.1111/j.1755-9294.2008.00008.x.

11. Waldron A. Odontogenic cysts and tumours. In: Neville B, editor. Oral and maxillofacial pathology. 2nd ed. Philadelphia, PA, USA: WB Saunders; 2002. p. 631-632.

12. Ashkenazi M, Greenberg BP, Chodik G, et al. Postoperative prognosis of unerupted teeth after removal of supernumerary teeth or odontomas. Am J Orthod Dentofac Orthop 2007;131(5):614-619. DOI: 10.1016/j.ajodo.2005.09.032.

13. Liu JK, Hsiao CK, Chen HA, et al. Orthodontic correction of a mandibular first molar deeply impacted by an odontoma: a case report. Quintessence Int (Berl) 1997;28(6):381-385. Available at: https://www.quintessence-publishing.com/deu/de/journal/ quintessence-international/1997/06.

14. Bereket C, Çakir-Özkan N, Şener I, et al. Complex and compound odontomas: analysis of 69 cases and a rare case of erupted compound odontoma. Niger J Clin Pract 2015;18(6):726-730. DOI: 10.4103/ 1119-3077.154209.

15. Hisatomi M, Asaumi Jl, Konouchi $\mathrm{H}$, et al. A case of complex odontoma associated with an impacted lower deciduous second molar and analysis of the 107 odontomas. Oral Dis 2002;8(2):100-105. DOI: 10.1034/j.1601-0825.2002.1c778.x.

16. Morning P. Impacted teeth in relation to odontomas. Int J Oral Surg 1980;9(2):81-91. DOI: 10.1016/S0300-9785(80)80043-3.

17. Harijadi A. Early removal of odontoma resulting in spontaneous eruption of the impacted teeth. Dent J 2010;43(2):76. DOI: 10.20473/ j.djmkg.v43.i2.p76-80. 